\title{
Generated paths of an autocatalytic set of a secondary system of a pressurized water reactor
}

\author{
Zainab Mahamud a, Tahir Ahmad a,b,* \\ a Department of Mathematical Science, Faculty of Science, Universiti Teknologi Malaysia, 81310 Skudai, Johor, Malaysia \\ ${ }^{b}$ Centre for Sustainable Nanomaterials, Ibnu Sina Institute for Scientific and Industrial Research, Universiti Teknologi Malaysia, 81310 UTM Johor \\ Bahru, Johor, Malaysia \\ * Corresponding author: tahir@ibnusina.utm.my
}

\section{Article history}

Received 26 January 2017

Accepted 19 March 2017

\begin{abstract}
A graph is used to model pairwise relation between objects. In this paper, it is used to model secondary system of pressurized water reactor. The process is presented as a dynamic graph by integrating the concept of Autocatalytic Set (ACS). The graph is then transformed into an omega algebra whereby all the possible paths of the process are determined. Seven variables are identified to represent the nodes with twenty one links to indicate catalytic relations among these nodes. A programming code of $\mathrm{C}_{++}$ is developed for the identification of these 317 links.
\end{abstract}

Keywords: Pressurized Water Reactor, autocatalytic set, omega algebra

\section{INTRODUCTION}

A nuclear power plant is a power station that generates electricity using heat from nuclear reactions. Steam is produced from heated water to spin the large turbines that generate electricity. One type of a nuclear power plant is Pressurized Water Reactor (PWR) (Nelson, 1982). PWR contains two systems namely primary and secondary systems (Ahmet et al., 2001) as shown in Fig. 1.

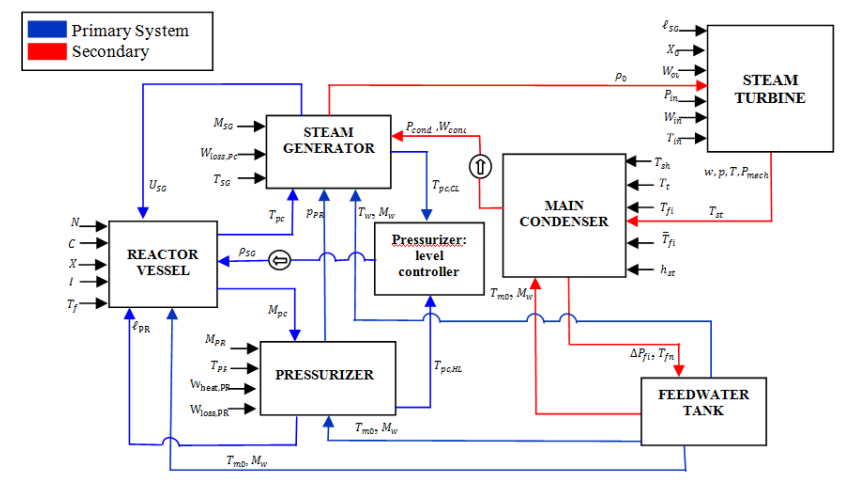

Fig. 1 Schematic diagram of a Pressurized Water Reactor (PWR) (Ashaari et al., 2015b).

Primary system of PWR consists of three main components namely reactor vessel, pressurizer and steam generator while for secondary system of PWR components are steam generator, main turbine and main condenser. Both systems operate simultaneously with a separate flow system. The steam generator is known as the bridge between the primary and secondary systems where it is a place for phase changes from water to steam (Ashaari et al., 2015a).

In this paper, we will focus on a secondary system of PWR. Most problems which occur in a secondary system are caused by the chemical compounds in the system (Ashaari et al., 2015b). He modelled the process using graph. The aim of this paper is to find all the possible paths in the secondary system of PWR given in Ashaari et al. (2015b).

\section{AUTOCATALYTIC SET}

The term catalysis was introduced in 1836 by Berzelius to explain various decomposition and transformation reactions (Hagen, 2015). An Autocatalytic Set (ACS) is a concept introduced in the context of catalytically interacting molecules by Kauffman (1971), Eigen (1971) and Rossler (1971). A chemical compound which is used to increase chemical reactions is known as a catalyst. An autocatalytic can be defined as a set of entities where the entities can be anything such as people, molecules or objects (Rossler, 1971). ACS are considered to be fundamental to the origin of life (Hordijk, 2015).

In 1998, Jain and Krishna formalized ACS in terms of graph. An autocatalytic set is described by a directed graph with nodes represent species and the directed links represent catalytic interactions among them. A link from node $j$ to node $i$ indicates that species $j$ is a catalyst for $i$.

Definition 1 (Jain and Krishna, 1998)

An autocatalytic set is a subgraph, each of whose nodes has at least one incoming link from a node belonging to the same subgraph. 


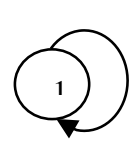

(a)

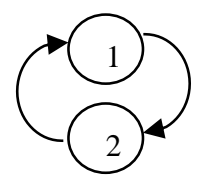

(b)

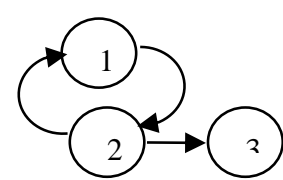

(c)
Fig. 2 Examples of Autocatalytic Set (ACS).

A graph with $n$ nodes is completely specified by an $n \times n$ matrix, $C=\left(C_{i j}\right)$ which is called the adjacency matrix of the graph. A graph is transformed to a square adjacency matrix, $C$ where its components are given as

$$
C_{i j}= \begin{cases}1 & \text { if }\left(v_{j}, v_{i}\right) \in E \\ 0 & \text { if }\left(v_{j}, v_{i}\right) \notin E\end{cases}
$$

By using (1), the adjacency matrices of the graphs in Fig. 2 are given as
(a) $[1]$
(b) $\left[\begin{array}{ll}0 & 1 \\ 1 & 0\end{array}\right]$
(c) $\left[\begin{array}{lll}0 & 1 & 0 \\ 1 & 0 & 0 \\ 0 & 1 & 0\end{array}\right]$

Fig. 3 Adjacency matrices.

\section{IMPLEMENTATION}

By using the concept of autocatalytic set, the secondary system of PWR can be represented as the dynamic graph. In 2015b, Ashaari et al. identified the chemical reactions that involve in that process of secondary system of PWR. Seven important compounds that are identified in the process are corrosion $\left(v_{1}\right)$, moderator $\left(v_{2}\right)$, sulphuric acid $\left(v_{3}\right)$, boric acid $\left(v_{4}\right)$, sodium hydroxide $\left(v_{5}\right)$, chlorides $\left(v_{6}\right)$ and nitrogen $\left(v_{7}\right)$. There are twenty one edges that represent catalytic relationship among these compounds.

$$
\begin{aligned}
E=\{ & \left(v_{2}, v_{1}\right),\left(v_{3}, v_{1}\right),\left(v_{4}, v_{1}\right),\left(v_{5}, v_{1}\right),\left(v_{6}, v_{1}\right),\left(v_{7}, v_{1}\right),\left(v_{1}, v_{2}\right), \\
& \left(v_{4}, v_{2}\right),\left(v_{7}, v_{2}\right),\left(v_{2}, v_{3}\right),\left(v_{5}, v_{3}\right),\left(v_{7}, v_{3}\right),\left(v_{2}, v_{4}\right),\left(v_{5}, v_{4}\right), \\
& \left.\left(v_{7}, v_{4}\right),\left(v_{2}, v_{5}\right),\left(v_{7}, v_{5}\right),\left(v_{2}, v_{6}\right),\left(v_{5}, v_{6}\right),\left(v_{7}, v_{6}\right),\left(v_{2}, v_{7}\right)\right\}
\end{aligned}
$$

The dynamic graph of the secondary system of PWR in Ashaari et al. (2015b) is given in Fig. 4.

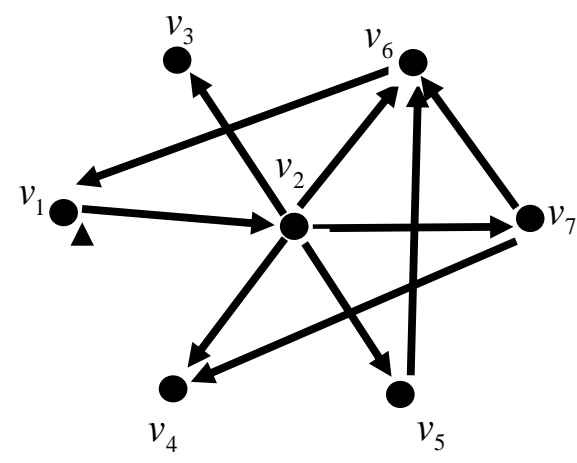

Fig. 4 Graph secondary system of PWR.
The adjacency matrix of the graph is represented as follows.

$$
C_{i j}=\left(\begin{array}{lllllll}
0 & 1 & 1 & 1 & 1 & 1 & 1 \\
1 & 0 & 0 & 1 & 0 & 0 & 1 \\
0 & 1 & 0 & 0 & 1 & 0 & 1 \\
0 & 1 & 0 & 0 & 1 & 0 & 1 \\
0 & 1 & 0 & 0 & 0 & 0 & 1 \\
0 & 1 & 0 & 0 & 1 & 0 & 1 \\
0 & 1 & 0 & 0 & 0 & 0 & 0
\end{array}\right)
$$

A path in a graph is a walk in which no vertices are repeated. For any pair of vertices with path of length one (adjacent), their catalytic relation is direct while for any path of length greater than one, the catalytic relation is indirect. Therefore, in the graph of the secondary system of PWR, there are direct and indirect catalytic relations. For example, moderator catalyst directly (length 1 ) in the production of corrosion, $\left(v_{2} \rightarrow v_{1}\right)$. Similarly the moderator can also catalyst indirectly (length 2 ) the production of corrosion via sulphuric acid, $\left(v_{2} \rightarrow v_{3} \rightarrow v_{1}\right)$.

Liew (2015) used omega algebra to model all the possible paths (direct and indirect catalytic relation) of a graph given in Tahir et al., 2010. The omega algebra is defined as follows:

Definition 2 (Liew, 2015)

An operation is a procedure that achieves a new element result from one of more input entity (ies). Given a set $M$, the $N$-ary algebraic operation on the set $M$ is expressed as $\omega_{n}: M_{1} \times M_{2} \times \ldots \times M_{n} \rightarrow M$.

That is to say, the $n$-ary or arity of a function or operation is the number of operands of the function. For some set $M, \omega$ is an operation and $n$ is its arity, $\omega_{n}: M^{n} \rightarrow M$. Hence, omega algebra ( $\Omega$-algebra) is a set by means of a defined system of operations $\Omega$ namely $\Omega$-algebra $=$ $\left\{\omega_{k} \mid k=0,1,2, \ldots, n\right\}$ which is defined on one primary set and is called one-sorted algebraic system. The concept of omega algebra can be viewed as a system of mapping such that;

$$
\begin{aligned}
& \omega_{2}: M \times M \rightarrow M \\
& \omega_{3}: M \times M \times M \rightarrow M \\
& \vdots \\
& \omega_{n}: \underbrace{M \times M \times \cdots \times M}_{n \text { times }} \rightarrow M
\end{aligned}
$$

The notation $\omega_{k\left(v_{i}, v_{j}\right)}$ represents " $v_{i}$ and $v_{j}$ have $k$-ary relation with $v_{i}$ catalyzed the production of $v_{j}$ through $k$-ary Cartesian product of $\omega$ operation. In other words, there exist some $v_{i}$ and $v_{j}$ such that $v_{i}$ and $v_{j}$ have $k$-ary catalytic relation in $\omega$-operation".

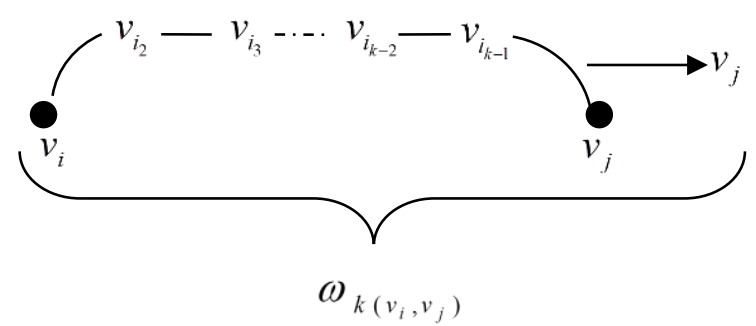

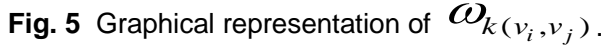


The * operation of omega algebra is used to represent the direct and indirect catalytic relation in secondary system of PWR.

1) Binary operation:

$\omega_{2}: *_{2}: V \times V \rightarrow V$ such that there exist some $v_{i}, v_{j} \in V$ and $v_{i} *_{2} v_{j}=v_{j} \in V \cdot$ (two vertices with length 1$)$

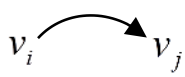

2) Ternary operation:

$\omega_{3}: *_{3}: V \times V \times V \rightarrow V$ such that there exist some

$v_{i}, v_{j}, v_{k} \in V$ and $v_{i} *_{3} v_{k}=v_{k} \in V$ through $v_{j}$. (three vertices with length 2)

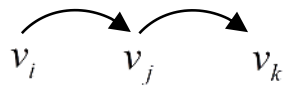

3) Quaternary operation:

$\omega_{4}: *_{4}: V \times V \times V \times V \rightarrow V$ such that there exist some

$v_{i}, v_{j}, v_{k}, v_{l} \in V$ and $v_{i} *_{4} v_{l}=v_{l} \in V$ through $v_{j}$ and $v_{k}$.

(four vertices with length 3 )

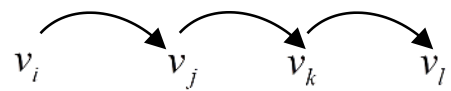

4) Quinary operation:

$\omega_{5}:{ }_{5}: V \times V \times V \times V \times V \rightarrow V$ such that there exist some

$v_{i}, v_{j}, v_{k}, v_{l}, v_{m} \in V$ and $v_{i} *_{5} v_{m}=v_{m} \in V$ through $v_{j}, v_{k}$

and $v_{l}$ (five vertices with length 4 )

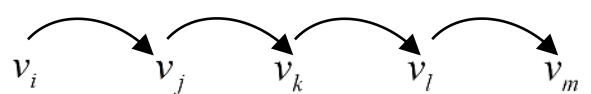

5) Senary operation:

$\omega_{6}:{ }_{6}: V \times V \times V \times V \times V \times V \rightarrow V$ such that there exist some

$v_{i}, v_{j}, v_{k}, v_{l}, v_{m}, v_{n} \in V$ and $v_{i} *_{6} v_{n}=v_{n} \in V$ through $v_{j}$,

$v_{k}, v_{l}$ and $v_{m}$ (six vertices with length 5)

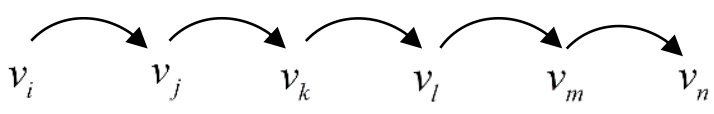

6) Septenary operation:

$\omega_{7}: *_{7}: V \times V \times V \times V \times V \times V \times V \rightarrow V$ such that there exist

some $v_{i}, v_{j}, v_{k}, v_{l}, v_{m}, v_{n}, v_{o} \in V$ and $v_{i} *_{7} v_{o}=v_{o} \in V$

through $v_{j}, v_{k}, v_{l} v_{m}$ and $v_{n}$. (seven vertices with length 6)

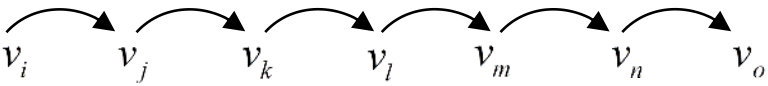

Therefore, the set of omega algebra of the secondary system of PWR is $\Omega_{G}=\left\{*_{2}, *_{3}, *_{4}, *_{5}, *_{6}, *_{7}\right\}$. In order to find all the possible paths, a programming code of $\mathrm{C}++$ language programming is developed.

\section{RESULTS AND DISCUSSION}

There are a total of 317 possible paths for the secondary system of pressurized water reactor.

Table 1 The length of the paths with its number of paths.

\begin{tabular}{cc}
\hline Length & Number of Paths \\
\hline $1\left(*_{2}\right)$ & 21 \\
$2\left(*_{3}\right)$ & 50 \\
$3\left(*_{4}\right)$ & 93 \\
$4\left(\begin{array}{c}* \\
*_{5}\end{array}\right)$ & 106 \\
$5\left(*_{6}\right)$ & 47 \\
$6\left(*_{7}\right)$ & 0 \\
\hline Total & 317 \\
\hline
\end{tabular}

Thus, the maximum number of length for the secondary system of pressurized water reactor is 5 .

The possible paths which connect every possible pair of vertices in the process are length less than seven. These links are summarized in Table 2 and Table 3 . The vertices in first column and the first row represent the elements which involve in the operations. The element in the first column catalysts the production of the element in the first row, direct or indirectly.

Table 2 Catalytic relation between vertices in secondary system of PWR for $i=\{1,2,3\}$ and $j=\{1,2,3,4,5,6,7\}$.

\begin{tabular}{|c|c|c|c|}
\hline $\begin{array}{l}\text { Catalytic } \\
\text { relation }\end{array}$ & $v_{1}$ & $v_{2}$ & $v_{3}$ \\
\hline$v_{1}$ & $*_{3}, *_{4}, *_{5}, *_{6}$ & $*_{2}$ & $*_{3}, *_{4}, *_{5}$ \\
\hline$v_{2}$ & $*_{2}, *_{3}, *_{4}, *_{5}$ & $*_{3}, *_{4}, *_{5}, *_{6}$ & $*_{2}, *_{3}, *_{4}$ \\
\hline$v_{3}$ & $*_{2}$ & $*_{3}$ & $*_{4}, *_{5}, *_{6}$ \\
\hline$v_{4}$ & $*_{2}, *_{3}, *_{4}, *_{5}, *_{6}$ & $*_{2}, *_{3}$ & $*_{3}, *_{4}, *_{5}, *_{6}$ \\
\hline$v_{5}$ & $*_{2}, *_{3}, *_{4}, *_{5}, *_{6}$ & $*_{3}, *_{4}$ & $*_{2}, *_{4}, *_{5}, *_{6}$ \\
\hline$v_{6}$ & $*_{2}$ & $*_{3}$ & $*_{4}, *_{5}, *_{6}$ \\
\hline$v_{7}$ & $*_{2}, *_{3}, *_{4}, *_{5}, *_{6}$ & $*_{2}, *_{3}, *_{4}, *_{5}$ & $*_{2}, *_{3}, *_{4}, *_{5}, *_{6}$ \\
\hline
\end{tabular}


Table 3 Catalytic relation between vertices in secondary system of PWR for $i=\{4,5,6,7\}$ and $j=\{1,2,3,4,5,6,7\}$.

\begin{tabular}{|c|c|c|c|c|}
\hline $\begin{array}{l}\text { Catalytic } \\
\text { relation }\end{array}$ & $v_{4}$ & $v_{5}$ & $v_{6}$ & $v_{7}$ \\
\hline$v_{1}$ & $*_{3}, *_{4}, *_{5}$ & $*_{3}, *_{4}$ & $*_{3}, *_{4}, *_{5}$ & $*_{3}$ \\
\hline$v_{2}$ & $*_{2}, *_{3}, *_{4}$ & $*_{2}, *_{3}$ & $*_{2}, *_{3}, *_{4}$ & $*_{2}$ \\
\hline$v_{3}$ & $*_{4}, *_{5}, *_{6}$ & $*_{4}, *_{5}$ & $*_{4}, *_{5}, *_{6}$ & $*_{4}$ \\
\hline$v_{4}$ & $*_{3}, *_{4}, *_{5}, *_{6}$ & $*_{3}, *_{4}, *_{5}$ & $*_{3}, *_{4}, *_{5}, *_{6}$ & $*_{3}, *_{4}$ \\
\hline$v_{5}$ & $*_{2}, *_{4}, *_{5}, *_{6}$ & $*_{4},{ }_{5}, *_{6}$ & $*_{2}, *_{4}, *_{5}, *_{6}$ & $*_{4}, *_{5}$ \\
\hline$v_{6}$ & $*_{4}, *_{5}, *_{6}$ & $*_{4}, *_{5}$ & $*_{4}, *_{5}, *_{6}$ & $*_{4}$ \\
\hline$v_{7}$ & $*_{2}, *_{3}, *_{4}, *_{5}, *_{6}$ & $*_{2}, *_{3}, *_{4}, *_{5}$ & $*_{2}, *_{3}, *_{4}, *_{5}, *_{6}$ & $*_{3},{ }_{4},{ }_{5},{ }_{6}$ \\
\hline
\end{tabular}

Table 4 presents the pair of vertices with their possible number of paths.

Table 4 The possible number of paths in secondary system of PWR for $i, j=\{1,2,3,4,5,6,7\}$.

\begin{tabular}{|c|c|c|c|c|c|c|c|c|}
\hline $\begin{array}{c}\text { Catalytic } \\
\text { relation }\end{array}$ & $v_{1}$ & $v_{2}$ & $v_{3}$ & $v_{4}$ & $v_{5}$ & $v_{6}$ & $v_{7}$ & Total \\
\hline$v_{1}$ & 16 & 1 & 4 & 4 & 2 & 4 & 1 & 32 \\
\hline$v_{2}$ & 16 & 21 & 4 & 4 & 2 & 4 & 1 & 52 \\
\hline$v_{3}$ & 1 & 1 & 4 & 4 & 2 & 4 & 1 & 17 \\
\hline$v_{4}$ & 13 & 2 & 8 & 8 & 4 & 8 & 2 & 45 \\
\hline$v_{5}$ & 10 & 5 & 9 & 7 & 10 & 9 & 5 & 55 \\
\hline$v_{6}$ & 1 & 1 & 4 & 4 & 2 & 4 & 1 & 17 \\
\hline$v_{7}$ & 25 & 11 & 16 & 13 & 7 & 16 & 11 & 99 \\
\hline Total & 82 & 42 & 49 & 44 & 29 & 49 & 22 & 317 \\
\hline
\end{tabular}

Therefore, all the compounds play vital roles in the secondary system of PWR. Nitrogen $\left(v_{7}\right)$ is the most catalyst compound with a total of ninety nine paths and corrosion $\left(v_{1}\right)$ is the most abundant produced compound with a total of eighty two paths.

\section{CONCLUSION}

There are a total of 317 possible paths for secondary system of the pressurized water reactor given in Ashaari et al. (2015b).

\section{ACKNOWLEDGEMENT}

This work has been supported by FRGS Grant, Vote No 4F756.

\section{REFERENCES}

Ahmet, D. and Hasbi, Y. (2001). Exergy analysis of a pressurized-water reactor nuclear-power plant. Applied Energy. 69(1), 39-57.

Ashaari, A., Tahir, A., Mustaffa, S., and Nazira, O. (2015a). Modeling steam generator system of pressurized water reactor using fuzzy state space. International Journal of Pure and Applied Mathematics. 103(1) 123-132.

Ashaari, A., Tahir, A., Mustaffa, S., Wan Munirah, W. M., and Nazira, O. (2015b). Graph representation for secondary System of pressurized water reactor with autocatalytic set approach. Journal of Mathematics and Statistics. 11(4), 107-112.

Eigen, M. (1971). Selforganization of matter and the evolution of biological macromolecules. Naturwissenchaften. 58(10), 465-523.

Hagen, J. (2015). Industrial catalysis: a practical approach. John Wiley \& Sons.

Hordijk, W., Smith, J. I., and Steel, M. (2015). Algorithms for detecting and analysing autocatalytic sets. Algorithms for Molecular Biology, 10(1), 15.

Jain, S. and Krishna, S. (1998). Autocatalytic sets and the growth of complexity in an evolutionary model. Physical Review Letters. 81(25), 5684-5687.

Kauffman, S. A. (1971). Cellular homeostasis, epigenesist and replication in randomly aggregated macromolecular systems. Journal of Cybernetics. 1 , 71-96.

Liew, S. Y. (2015). Omega algebra model of clinical waste incineration process Doctor of Philosophy, Universiti Teknologi Malaysia (UTM), Skudai.

Nelson, W. R. (1982). REACTOR: An expert system for diagnosis and treatment of nuclear reactor accidents. Proceedings of the Second AAAI Conference on Artificial Intelligence (AAAI'82). August 18-20, 1982. Pittsburgh, Pennsylvania: AAAI Press. 296-301.

Rossler, O.E. (1971). A system theoretic model of biogenesis. Z. Naturforschung. 26(8), 741-746.

Tahir, A., Sabariah, B. and Khairil, A. A. (2010). Modeling a clinical incineration process using fuzzy autocatalytic set. Journal of Mathematical Chemistry. 47(4), 1263-1273. 